physics $/ 9705040$

\title{
Lowest-energy representations of non-centrally extended diffeomorphism algebras
}

\author{
T. A. Larsson \\ Vanadisvägen 29 \\ S-113 23 Stockholm, Sweden \\ email: tal@hdd.se
}

\begin{abstract}
We describe a class of non-central extensions of the diffeomorphism algebra in $N$-dimensional spacetime, and construct lowest-energy modules thereof, thus generalizing work of Eswara-Rao and Moody. There is one representation for each representation of $\operatorname{Vir} \widetilde{\ltimes}_{k_{0}} \widehat{g l(N)}$ (an extension of the semi-direct product). Similar modules are constructed for gauge algebras.
\end{abstract}

\section{Introduction}

Let $\operatorname{diff}(N)$ denote the diffeomorphism algebra in $N$-dimensional spacetime. In a significant paper, Eswara-Rao and Moody constructed the first interesting lowest-energy representations of a non-central extension thereof [5], but they failed to explicitly describe the extension (except for the "spatial" subalgebra generated by time-independent vector fields). In the present paper, a four-parameter non-central extension $\widetilde{\operatorname{diff}}\left(N ; c_{1}, c_{2}, c_{3}, c_{4}\right)$ is described explicitly, and a realization of this algebra is constructed for each 
representation of $\operatorname{Vir} \widetilde{\ltimes}_{k_{0}} \widehat{g l(N)}$ (an extension of $\left.\operatorname{Vir} \ltimes \widehat{g l(N)}\right)$. The representations in [5] are recovered (in a Fourier basis) by picking a particular vertex operator module for $V i r$ and the trivial module for $\widehat{g l(N)}$. Thus, my results are related to theirs in about the same way as tensor densitites are related to functions. Similar results also hold for the algebra of gauge transformations on spacetime; special cases were previously found by [4, 6, 10]. A supersymmetric generalization of the present work can be found on the web 9 .

After this work was completed I became aware of related references [1], 2].

\section{Extension}

Let $\xi=\xi^{\mu} \partial_{\mu}$ be a vector field and $\mathcal{L}_{\xi}$ the Lie derivative. Greek indices $\mu, \nu=$ $0,1, . ., N-1$ label spacetime coordinates and the summation convention is used. The diffeomorphism algebra (algebra of vector fields, Witt algebra) $\operatorname{diff}(N)$ is generated by Lie derivatives satisfying $\left[\mathcal{L}_{\xi}, \mathcal{L}_{\eta}\right]=\mathcal{L}_{[\xi, \eta]}$. Define two families of operators $S_{n}^{\nu_{1} . . \nu_{n}}\left(g_{\nu_{1} . . \nu_{n}}\right)$ and $R_{n}^{\rho \mid \nu_{1} . . \nu_{n}}\left(h_{\rho \mid \nu_{1} . . \nu_{n}}\right)$, where $g_{\nu_{1} . . \nu_{n}}$ and $h_{\rho \mid \nu_{1} . . \nu_{n}}$ are arbitrary functions on spacetime. The operators are linear in the arguments and totally symmetric in the indices $\nu_{1} . . \nu_{n}$. The following relations (2.1 2.3) define a Lie algebra extension of diff $(N)$, denoted by $\widetilde{\operatorname{diff}}\left(N ; c_{1}, c_{2}, c_{3}, c_{4}\right)$.

$$
\begin{aligned}
& {\left[\mathcal{L}_{\xi}, S_{n}^{\nu_{1} . . \nu_{n}}\left(g_{\nu_{1} . . \nu_{n}}\right)\right]=S_{n}^{\nu_{1} . . \nu_{n}}\left(\xi^{\mu} \partial_{\mu} g_{\nu_{1} . . \nu_{n}}+\sum_{j=1}^{n} \partial_{\nu_{j}} \xi^{\mu} g_{\nu_{1} . . \mu . . \nu_{n}}\right)} \\
& \quad-(n-1) S_{n+1}^{\mu \nu_{1} . . \nu_{n}}\left(\partial_{\mu} \xi^{0} g_{\nu_{1} . . \nu_{n}}\right), \\
& S_{1}^{\nu}\left(\partial_{\nu} f\right) \equiv 0, \\
& S_{n+1}^{0 \nu_{1} . . \nu_{n}}\left(g_{\nu_{1} . . \nu_{n}}\right)=S_{n}^{\nu_{1} . . \nu_{n}}\left(g_{\nu_{1} . . \nu_{n}}\right), \\
& S_{0}(f)=\frac{1}{2 \pi i} \int d t f(t), \quad \text { if } f(t) \text { depends on time only, } \\
& {\left[\mathcal{L}_{\xi}, R_{n}^{\rho \mid \nu_{1} . . \nu_{n}}\left(h_{\rho \mid \nu_{1} . . \nu_{n}}\right)\right]=R_{n}^{\rho \mid \nu_{1} . . \nu_{n}}\left(\xi^{\mu} \partial_{\mu} h_{\rho \mid \nu_{1} . . \nu_{n}}+\partial_{\rho} \xi^{\mu} h_{\mu \mid \nu_{1} . . \nu_{n}}\right.} \\
& \left.\quad+\sum_{j=1}^{n} \partial_{\nu_{j}} \xi^{\mu} h_{\rho \mid \nu_{1} . . \mu . . \nu_{n}}\right) \\
& \quad-(n+1) R_{n+1}^{\rho \mid \mu \nu_{1} . . \nu_{n}}\left(\partial_{\mu} \xi^{0} h_{\rho \mid \nu_{1} . . \nu_{n}}\right)-R_{n+1}^{\rho \mid \mu \nu_{1} . . \nu_{n}}\left(\partial_{\rho} \xi^{0} h_{\mu \mid \nu_{1} . . \nu_{n}}\right) \\
& \quad+S_{n+2}^{\rho \sigma \nu_{1} . . \nu_{n}}\left(\partial_{\rho} \partial_{\sigma} \xi^{\mu} h_{\mu \mid \nu_{1} . . \nu_{n}}\right)-S_{n+3}^{\rho \sigma \mu \nu_{1} . . \nu_{n}}\left(\partial_{\rho} \partial_{\sigma} \xi^{0} h_{\mu \mid \nu_{1} . . \nu_{n}}\right),
\end{aligned}
$$




$$
\begin{aligned}
& S_{n+1}^{\mu \nu_{1} . \nu_{n}}\left(\partial_{\mu} h_{\nu_{1} . . \nu_{n}}\right)+\sum_{j=1}^{n} R_{n-1}^{\nu_{j} \mid \nu_{1} . \check{\nu}_{j} . . \nu_{n}}\left(h_{\nu_{1} . . \nu_{n}}\right) \equiv 0, \\
& \quad \text { where } h_{\nu_{1} . . \nu_{n}} \text { is symmetric in } \nu_{1} . . \nu_{n} . \\
& R_{n+1}^{\rho \mid 0 \nu_{1} . . \nu_{n}}\left(h_{\rho \mid \nu_{1} . . \nu_{n}}\right) \equiv R_{n}^{\rho \mid \nu_{1} . . \nu_{n}}\left(h_{\rho \mid \nu_{1} . . \nu_{n}}\right), \\
& R_{n}^{0 \mid \nu_{1} . . \nu_{n}}\left(h_{\nu_{1} . . \nu_{n}}\right) \equiv 0, \\
& \left.\quad \mathcal{L}_{\xi}, \mathcal{L}_{\eta}\right]=\mathcal{L}_{[\xi, \eta]}+c_{1} S_{1}^{\rho}\left(\partial_{\rho} \partial_{\nu} \xi^{\mu} \partial_{\mu} \eta^{\nu}\right)+c_{2} S_{1}^{\rho}\left(\partial_{\rho} \partial_{\mu} \xi^{\mu} \partial_{\nu} \eta^{\nu}\right) \\
& \quad+c_{3}\left(R_{1}^{\mu \mid \nu}\left(\partial_{\mu} \xi^{0} \partial_{\nu} \eta^{0}\right)+S_{3}^{\mu \nu \rho}\left(\partial_{\rho} \partial_{\mu} \xi^{0} \partial_{\nu} \eta^{0}\right)\right) \\
& \quad+\frac{c_{4}}{2} S_{2}^{\rho \sigma}\left(\partial_{\rho} \eta^{0} \partial_{\sigma} \partial_{\mu} \xi^{\mu}-\partial_{\rho} \xi^{0} \partial_{\sigma} \partial_{\nu} \eta^{\nu}\right) \\
& \quad+a_{1}\left(S_{2}^{\rho \sigma}\left(\partial_{\rho} \partial_{\sigma} \xi^{\mu} \partial_{\mu} \eta^{0}-\partial_{\rho} \partial_{\sigma} \eta^{\nu} \partial_{\nu} \xi^{0}\right)\right. \\
& \left.\quad-S_{3}^{\rho \mu \nu}\left(\partial_{\rho} \partial_{\mu} \xi^{0} \partial_{\nu} \eta^{0}-\partial_{\rho} \partial_{\nu} \eta^{0} \partial_{\mu} \xi^{0}\right)\right) \\
& \quad-a_{2} S_{1}^{\rho}\left(\partial_{\rho} \xi^{0} \eta^{0}\right)+a_{3} S_{1}^{\rho}\left(\partial_{\rho} \eta^{0} \partial_{\mu} \xi^{\mu}-\partial_{\rho} \xi^{0} \partial_{\nu} \eta^{\nu}\right), \\
& {\left[S_{m}^{\mu_{1} . . \mu_{m}}\left(g_{\mu_{1} . . \mu_{m}}\right), S_{n}^{\nu_{1} . . \nu_{n}}\left(h_{\nu_{1} . . \nu_{n}}\right)\right]=\left[S_{m}^{\mu_{1} . . \mu_{m}}\left(g_{\mu_{1} . . \mu_{m}}\right), R_{n}^{\rho \mid \nu_{1} . . \nu_{n}}\left(h_{\rho \mid \nu_{1} . . \nu_{n}}\right)\right]} \\
& =\left[R_{m}^{\rho \mid \mu_{1} . . \mu_{m}}\left(h_{\rho \mid \mu_{1} . . \mu_{m}}\right), R_{n}^{\sigma \mid \nu_{1} . . \nu_{n}}\left(h_{\sigma \mid \nu_{1} . . \nu_{n}}\right)\right]=0 .
\end{aligned}
$$

Verification of all Jacobi identities is straightforward; that these equations define a Lie algebra also follows from the explicit realization in theorem 3.1 below. Indeed, the extensions were discovered by working out which algebra is generated by (3.6). The extensions $a_{1}-a_{3}$ (cocycles are labelled by the factors multiplying them) are cohomologically trivial and may be eliminated by the redefinition

$$
\mathcal{L}_{\xi}^{\prime}=\mathcal{L}_{\xi}+a_{1} S_{2}^{\mu \nu}\left(\partial_{\mu} \partial_{\nu} \xi^{0}\right)+\frac{a_{2}}{2} S_{0}\left(\xi^{0}\right)+a_{3} S_{0}\left(\partial_{\mu} \xi^{\mu}\right)
$$

The remaining extensions are non-trivial, which is most easily seen by restricting to the "temporal" subalgebra generated by vector fields on the line $x^{\mu}=t \delta_{0}^{\mu}$; it is a Virasoro algebra with central charge $12\left(c_{1}+c_{2}+c_{3}+c_{4}\right)$. In particular, this is the whole story in one dimension, and hence (2.1 2.3) is a natural higher-dimensional generalization of the Virasoro algebra. The extensions $c_{1}$ and $c_{2}$ were first described by Eswara-Rao and Moody [5] and myself [7], 8], respectively, while $c_{3}$ and $c_{4}$ are new. $S_{1}^{\rho}\left(g_{\rho}\right)$ is a linear operator acting on one-forms $g_{\rho} d x^{\rho}$, and as such it may be viewed as a closed one-chain on spacetime. One possibility is that it is an exact one-chain:

$$
S_{1}^{\rho}\left(g_{\rho}\right)=C^{\nu \rho}\left(\partial_{\nu} g_{\rho}\right)
$$




$$
\begin{aligned}
C^{\nu \mu}\left(j_{\mu \nu}\right) & =-C^{\mu \nu}\left(j_{\mu \nu}\right) \\
{\left[\mathcal{L}_{\xi}, C^{\nu \rho}\left(j_{\nu \rho}\right)\right] } & =C^{\nu \rho}\left(\xi^{\mu} \partial_{\mu} j_{\nu \rho}+\partial_{\nu} \xi^{\mu} j_{\mu \rho}+\partial_{\rho} \xi^{\mu} j_{\nu \mu}\right) .
\end{aligned}
$$

Dzhumadil'daev [3] has given an list of $\operatorname{diff}(N)$ extensions by irreducible modules, but it seems that the extension $c_{1}$ is missing; however, it is essentially $\psi_{1}^{W}-\psi_{3}^{W}+\psi_{4}^{W}$ in his notation. Moreover, $c_{2}$ is $\psi_{1}^{W}$ and $c_{1}$ and $c_{2}$ become $\psi_{4}^{W}$ and $\psi_{3}^{W}$ upon the substitution (2.5), respectively. The remaining two cocycles are not covered by his theorem, however, because they are extensions by reducible but indecomposable modules.

\section{Realization}

Consider the Heisenberg algebra generated by operators $q^{i}(s), p_{j}(t), s, t \in$ $S^{1}$, where latin indices $i, j=1, . ., N-1$ run over spatial coordinates only.

$$
\begin{aligned}
{\left[p_{j}(s), q^{i}(t)\right] } & =\delta_{j}^{i} \delta(s-t), \\
{\left[p_{i}(s), p_{j}(t)\right] } & =\left[q^{i}(s), q^{j}(t)\right]=0 .
\end{aligned}
$$

These operators can be expanded in a Fourier series; e.g.,

$$
p_{j}(t)=\sum_{n=-\infty}^{\infty} \hat{p}_{j}(n) \mathrm{e}^{i n t} .
$$

This algebra has a Fock module $\mathcal{F}$ ( $\mathbb{Z}$-graded by the frequency $n$ ) generated by finite strings in the non-negative Fourier modes of $q^{i}(t)$ and the positive modes of $p_{j}(t)$. Define time components by $q^{0}(t)=t$ and $p_{0}(t)=-\dot{q}^{i}(t) p_{i}(t)$; in an obvious notation, $q^{\mu}(t)=\left(t, q^{i}(t)\right)$, etc. The following relations hold.

$$
\begin{aligned}
& {\left[q^{\mu}(s), q^{\nu}(t)\right]=0} \\
& {\left[p_{\nu}(s), q^{\mu}(t)\right]=\left(\delta_{\nu}^{\mu}-\dot{q}^{\mu}(s) \delta_{\nu}^{0}\right) \delta(s-t)} \\
& {\left[p_{\mu}(s), p_{\nu}(t)\right]=\left(\delta_{\mu}^{0} p_{\nu}(s)+\delta_{\nu}^{0} p_{\mu}(t)\right) \dot{\delta}(s-t) .}
\end{aligned}
$$

Normal ordering is necessary to remove infinites and to obtain a well defined action of diffeomorphisms on $\mathcal{F}$. For any function of $q(t)$ and its derivatives, let

$$
: f(q(t), \dot{q}(t)) p_{j}(t): \equiv f(q(t), \dot{q}(t)) p_{j}^{\leq}(t)+p_{j}^{>}(t) f(q(t), \dot{q}(t))
$$


where $p_{j}^{>}(t)\left(p_{j}^{\leq}(t)\right)$ is the sum (3.2) over positive (non-positive) Fourier modes only. Let $L(s)$ and $T_{\nu}^{\mu}(t)$ generate the following algebra $V i r_{c} \widetilde{\ltimes}_{k_{0}}$ $\widehat{\operatorname{gl}(N)}_{k_{1}, k_{2}}$.

$$
\begin{aligned}
{[L(s), L(t)]=} & (L(s)+L(t)) \dot{\delta}(s-t)+\frac{c}{24 \pi i}(\dddot{\delta}(s-t)+\dot{\delta}(s-t)) \\
{\left[L(s), T_{\nu}^{\mu}(t)\right]=} & T_{\nu}^{\mu}(s) \dot{\delta}(s-t)+\frac{k_{0}}{4 \pi i} \delta_{\nu}^{\mu} \ddot{\delta}(s-t) \\
{\left[T_{\nu}^{\mu}(s), T_{\tau}^{\sigma}(t)\right]=} & \left(\delta_{\nu}^{\sigma} T_{\tau}^{\mu}(s)-\delta_{\tau}^{\mu} T_{\nu}^{\sigma}(s)\right) \delta(s-t) \\
& -\frac{1}{2 \pi i}\left(k_{1} \delta_{\tau}^{\mu} \delta_{\nu}^{\sigma}+k_{2} \delta_{\nu}^{\mu} \delta_{\tau}^{\sigma}\right) \dot{\delta}(s-t)
\end{aligned}
$$

Theorem 3.1 Under the conditions above, the following expressions

$$
\begin{aligned}
& \mathcal{L}_{\xi}=\int d t: \xi^{\mu}(q(t)) p_{\mu}(t):+\xi^{0}(q(t)) L(t)+\partial_{\nu} \xi^{\mu}(q(t)) T_{\mu}^{\nu}(t) \\
& \equiv \int d t: \xi^{i}(q(t)) p_{i}(t):-: \xi^{0}(q(t)) \dot{q}^{i}(t) p_{i}(t): \\
& \quad+\xi^{0}(q(t)) L(t)+\partial_{\nu} \xi^{\mu}(q(t)) T_{\mu}^{\nu}(t), \\
& S_{n}^{\nu_{1} . . \nu_{n}}\left(g_{\nu_{1} . . \nu_{n}}\right)=\frac{1}{2 \pi i} \int d t \dot{q}^{\nu_{1}}(t) \ldots \dot{q}^{\nu_{n}}(t) g_{\nu_{1} . . \nu_{n}}(q(t)), \\
& R_{n}^{\rho \mid \nu_{1} . . \nu_{n}}\left(h_{\rho \mid \nu_{1} . . \nu_{n}}\right)=\frac{1}{2 \pi i} \int d t \ddot{q}^{\rho}(t) \dot{q}^{\nu_{1}}(t) \ldots \dot{q}^{\nu_{n}}(t) h_{\rho \mid \nu_{1} . . \nu_{n}}(q(t)),
\end{aligned}
$$

realize the Lie algebra $\widetilde{\operatorname{diff}}\left(N ; 1+k_{1}, k_{2},-2+(c+2 N-2) / 12,1+k_{0}\right)$, while the cohomologically trivial parameters are $a_{1}=-1, a_{2}=(c+2 N-2) / 12$, $a_{3}=i / 2$.

The proof is deferred to the appendix. Consequently, this algebra acts on $\mathcal{F} \otimes \mathcal{M}$ for every $V i r_{c} \widetilde{\ltimes}_{k_{0}} \widehat{g l(N)}_{k_{1}, k_{2}}$ module $\mathcal{M}$. It should be stressed that this action is manifestly well defined, at least for the subalgebra of vector fields that are polynomial in the spatial coordinates and a Fourier polynomial in $x^{0}$, because finiteness is preserved when all operators in (3.6) act on finite strings in non-negative Fourier modes in that case. The Hamiltonian

$$
\mathcal{L}_{-i \partial_{0}}=-i \int d t\left(-: \dot{q}^{i}(t) p_{i}(t):+L(t)\right)
$$


is the operator responsible for computing the $\mathbb{Z}$-grading.

In the absense of normal ordering and central charges in (3.5), (3.6) yields a proper realization of $\operatorname{dif} f(N)$. The higher-dimensional analogue of a primary field depends on five parameters $\lambda, w$ (defined up to an integer), $\kappa, p$, and $q$ :

$$
\begin{aligned}
& {\left[\mathcal{L}_{\xi}, \phi_{\tau_{1} . . \tau_{q}}^{\sigma_{1} . \sigma_{p}}(t)\right]=-\xi^{0}(q(t)) \dot{\phi}_{\tau_{1} . . . \tau_{q}}^{\sigma_{1} . \sigma_{p}}(t)-\lambda \dot{\xi}^{0}(q(t)) \phi_{\tau_{1} . . \tau_{q}}^{\sigma_{1} . \sigma_{p}}(t)} \\
& \quad+i w \xi^{0}(q(t)) \phi_{\tau_{1} . . . \tau_{q}}^{\sigma_{1} . \sigma_{p}}(t)-\kappa \partial_{\mu} \xi^{\mu}(q(t)) \phi_{\tau_{1} . . \tau_{q}}^{\sigma_{1} . \sigma_{p}}(t) \\
& \quad+\sum_{i=1}^{p} \partial_{\mu} \xi^{\sigma_{i}}(q(t)) \phi_{\tau_{1} . . \tau_{q}}^{\sigma_{1} . . . \sigma_{p}}(t)-\sum_{j=1}^{q} \partial_{\tau_{j}} \xi^{\mu}(q(t)) \phi_{\tau_{1} . . . . . . \tau_{q}}^{\sigma_{1} \ldots \sigma_{p}}(t)
\end{aligned}
$$

where $\left[\mathcal{L}_{\xi}, q^{\mu}(t)\right]=\xi^{\mu}(q(t))-\dot{q}^{\mu}(t) \xi^{0}(q(t))$.

The result of Eswara-Rao and Moody [5] is recovered as follows: they work in a Fourier basis on the torus, and denote $q^{i}(t)=\delta_{i}(z)$ and $p_{j}(t)=$ $d_{j}(z)$, where $z=\exp (i t)$. A standard vertex operator realization for the Virasoro generator $L(t)$ was given, based on the remaining roots $\alpha_{p}$, but they missed the appearance of $\widehat{g l(N)}$. Consequently, $T_{\nu}^{\mu}(t)=0$ and $k_{0}=$ $k_{1}=k_{2}=0$ in their work.

\section{Gauge algebras}

Consider the gauge algebra $\operatorname{map}(N, \mathfrak{g})$, i.e. maps from $N$-dimensional spacetime to a finite-dimensional Lie algebra $\mathfrak{g}$, where $\mathfrak{g}$ has basis $J^{a}$, structure constants $f^{a b}{ }_{c}$, and Killing metric $\delta^{a b}$. Define constants $g^{a}$ and $g^{\prime a}$ satisfying $f^{a b}{ }_{c} g^{c}=f^{a b}{ }_{c} g^{\prime c}=0$. Clearly, $g^{a}=g^{\prime a}=0$ if $J^{a} \in[\mathfrak{g}, \mathfrak{g}]$, but they may be non-zero on abelian factors. Let $X=X_{a}(x) J^{a}, x \in \mathbb{R}^{N}$ be a $\mathfrak{g}$-valued function and define $[X, Y]_{c}=i f{ }_{c}^{a b} X_{a} Y_{b}$. diff $(N) \ltimes \operatorname{map}(N, \mathfrak{g})$ has the non-central extension $\widetilde{\operatorname{diff} f}\left(N ; c_{1}, c_{2}, c_{3}, c_{4}\right) \widetilde{\ltimes}_{g, g^{\prime}} \widetilde{\text { map }}(N, \mathfrak{g} ; k)$, with brackets

$$
\begin{aligned}
{\left[\mathcal{J}_{X}, \mathcal{J}_{Y}\right] } & =\mathcal{J}_{[X, Y]}-k \delta^{a b} S_{1}^{\rho}\left(\partial_{\rho} X_{a} Y_{b}\right), \\
{\left[\mathcal{L}_{\xi}, \mathcal{J}_{X}\right] } & =\mathcal{J}_{\xi^{\mu}} \partial_{\mu} X-g^{a} S_{2}^{\mu \nu}\left(\partial_{\mu} \xi^{0} \partial_{\nu} X_{a}\right)-g^{\prime a} S_{1}^{\rho}\left(\partial_{\rho} \partial_{\mu} \xi^{\mu} X_{a}\right), \\
{\left[\mathcal{J}_{X}, S_{n}^{\nu_{1} . \nu_{n}}\left(g_{\nu_{1} . . \nu_{n}}\right)\right] } & =\left[\mathcal{J}_{X}, R_{n}^{\rho \mid \nu_{1} . . \nu_{n}}\left(h_{\rho \mid \nu_{1} . . \nu_{n}}\right)\right]=0,
\end{aligned}
$$

in addition to (2.3). Let $J^{a}(t), t \in S^{1}$, generate the Kac-Moody algebra $\widehat{\mathfrak{g}}_{k}$. Consider the algebra $\operatorname{Vir}_{c} \widetilde{\ltimes}_{k_{0}, g}\left(\widehat{g l(N)}_{k_{1}, k_{2}} \widetilde{\oplus}_{g^{\prime}} \widehat{\mathfrak{g}}_{k}\right)$, with brackets (3.5) and

$$
\left[J^{a}(s), J^{b}(t)\right]=i f_{c}^{a b} J^{c}(s) \delta(s-t)+\frac{k}{2 \pi i} \delta^{a b} \dot{\delta}(s-t)
$$




$$
\begin{aligned}
{\left[T_{\nu}^{\mu}(s), J^{a}(t)\right] } & =\frac{g^{\prime a}}{2 \pi i} \delta_{\nu}^{\mu} \dot{\delta}(s-t), \\
{\left[L(s), J^{a}(t)\right] } & =J^{a}(s) \dot{\delta}(s-t)+\frac{g^{a}}{2 \pi i} \ddot{\delta}(s-t) .
\end{aligned}
$$

Then

$$
\mathcal{J}_{X}=\int d t X_{a}(q(t)) J^{a}(t)
$$

yields a realization of $\widetilde{\operatorname{map}}(N, \mathfrak{g} ; k)$, with the intertwining action of $\widetilde{\operatorname{diff}}(N$; $\left.c_{1}, c_{2}, c_{3}, c_{4}\right)$ described above, and the parameters $k, g^{a}$ and $g^{\prime a}$ in (4.1) and (4.2) agree.

\section{A Proof of theorem 3.1}

We first prove that in absense of normal ordering, (3.6) defines a proper realization of $\operatorname{dif} f(N)$. The operators $\tilde{p}_{\nu}(t)=p_{\nu}(t)+\delta_{\nu}^{0} L(t)$ satisfy relations (3.3) and also

$$
\left[\tilde{p}_{\mu}(s), T_{\sigma}^{\nu}(t)\right]=\delta_{\mu}^{0} T_{\sigma}^{\nu}(s) \dot{\delta}(s-t) .
$$

Introduce the abbreviated notation $\xi^{\mu}(t) \equiv \xi^{\mu}(q(t))$. Now,

$$
\begin{aligned}
{\left[\mathcal{L}_{\xi}, \mathcal{L}_{\eta}\right]=} & \iint d s d t\left[\xi^{\mu}(s) \tilde{p}_{\mu}(s)+\partial_{\sigma} \xi^{\mu}(s) T_{\mu}^{\sigma}(s), \eta^{\nu}(t) \tilde{p}_{\nu}(t)+\partial_{\tau} \eta^{\nu}(t) T_{\nu}^{\tau}(t)\right] \\
= & \iint d s d t \xi^{\mu}(s)\left\{\partial_{\rho} \eta^{\nu}(t)\left(\delta_{\mu}^{\rho}-\delta_{\mu}^{0} \dot{q}^{\rho}(s)\right) \delta(s-t) \tilde{p}_{\nu}(t)\right. \\
& \left.+\eta^{\nu}(t) \delta_{\mu}^{0} \tilde{p}_{\nu}(s) \dot{\delta}(s-t)\right\} \\
& +\xi^{\mu}(s)\left\{\partial_{\rho} \partial_{\tau} \eta^{\nu}(t)\left(\delta_{\mu}^{\rho}-\delta_{\mu}^{0} \dot{q}^{\rho}(s)\right) \delta(s-t) T_{\nu}^{\tau}(t)\right. \\
& \left.+\partial_{\tau} \eta^{\nu}(t) \delta_{\mu}^{0} T_{\nu}^{\tau}(s) \dot{\delta}(s-t)\right\} \\
& +\partial_{\sigma} \xi^{\mu}(s) \partial_{\tau} \eta^{\nu}(t) \delta_{\mu}^{\tau} T_{\nu}^{\sigma}(s) \delta(s-t)-\xi \leftrightarrow \eta
\end{aligned}
$$

where $\xi \leftrightarrow \eta$ stands for the same expression with $\xi$ and $\eta$ interchanged everywhere. Rewrite the terms proportial to the derivative of the delta function by noting that

$$
\iint d s d t f(s) g(t) \dot{\delta}(s-t)=\int f \dot{g}=-\int \dot{f} g .
$$


The function arguments were suppressed in the single integrals, because no confusion is possible. This leaves us with

$$
\begin{aligned}
& \int \xi^{\mu}\left(\partial_{\mu} \eta^{\nu}-\delta_{\mu}^{0} \dot{q}^{\rho} \partial_{\rho} \eta^{\nu}\right) \tilde{p}_{\nu}+\xi^{\mu} \dot{\eta}^{\nu} \delta_{\mu}^{0} \tilde{p}_{\nu} \\
& +\xi^{\mu} \partial_{\rho} \partial_{\tau} \eta^{\nu}\left(\delta_{\mu}^{\rho}-\delta_{\mu}^{0} \dot{q}^{\rho}\right) T_{\nu}^{\tau}+\xi^{\mu} \partial_{\tau} \dot{\eta}^{\nu} \delta_{\mu}^{0} T_{\nu}^{\tau}+\partial_{\sigma} \xi^{\mu} \partial_{\mu} \eta^{\nu} T_{\nu}^{\sigma}-\xi \leftrightarrow \eta \\
= & \int \xi^{\mu} \partial_{\mu} \eta^{\nu} \tilde{p}_{\nu}+\xi^{\mu} \partial_{\mu} \partial_{\tau} \eta^{\nu} T_{\nu}^{\tau}+\partial_{\sigma} \xi^{\mu} \partial_{\mu} \eta^{\nu} T_{\nu}^{\sigma}-\xi \leftrightarrow \eta \\
= & \int\left(\xi^{\mu} \partial_{\mu} \eta\right)^{\nu} \tilde{p}_{\nu}+\partial_{\tau}\left(\xi^{\mu} \partial_{\mu} \eta^{\nu}\right) T_{\nu}^{\tau}-\xi \leftrightarrow \eta=\mathcal{L}_{\xi^{\mu} \partial_{\mu} \eta}-\xi \leftrightarrow \eta,
\end{aligned}
$$

where we used that $\dot{\eta}^{\nu}=\dot{q}^{\rho} \partial_{\rho} \eta^{\nu}$. Hence $\left[\mathcal{L}_{\xi}, \mathcal{L}_{\eta}\right]=\mathcal{L}_{[\xi, \eta]}$, and it is clear that normal ordering must result in some abelian extension of $\operatorname{dif} f(N)$. We now proceed to calculate it.

Split the delta function into positive and negative energy parts.

$$
\delta^{>}(t)=\frac{1}{2 \pi} \sum_{m>0} \mathrm{e}^{-i m t}, \quad \delta^{\leq}(t)=\frac{1}{2 \pi} \sum_{m \leq 0} \mathrm{e}^{-i m t} .
$$

\section{Lemma A.1}

$$
\begin{array}{ll}
\text { i. } & \delta^{>}(t) \delta^{\leq}(-t)-\delta^{>}(-t) \delta^{\leq}(t)=-\frac{1}{2 \pi i} \dot{\delta}(t) \\
\text { ii. } & \delta^{>}(t) \dot{\delta}^{\leq}(-t)-\dot{\delta}^{>}(-t) \delta^{\leq}(t)=\frac{1}{4 \pi i}(\ddot{\delta}(t)+i \dot{\delta}(t)) \\
\text { iii. } & \dot{\delta}^{>}(t) \dot{\delta}^{\leq}(-t)-\dot{\delta}^{>}(-t) \dot{\delta}^{\leq}(t)=\frac{1}{12 \pi i}(\dddot{\delta}(t)+\dot{\delta}(t))
\end{array}
$$

Proof:

$$
\begin{array}{ll}
\text { i. } \quad & 4 \pi^{2} \cdot L H S=\sum_{m>0} \sum_{n \leq 0}\left(\mathrm{e}^{-i(m-n) t}-\mathrm{e}^{i(m-n) t}\right)=\sum_{k>0} \sum_{m=1}^{k}\left(\mathrm{e}^{-i k t}-\mathrm{e}^{i k t}\right) \\
& =\sum_{k>0} k\left(\mathrm{e}^{-i k t}-\mathrm{e}^{i k t}\right)=\sum_{k} k \mathrm{e}^{-i k t}=2 \pi i \dot{\delta}(t) \text { where } k=m-n . \\
\text { ii. } \quad & 4 \pi^{2} i \cdot L H S=\sum_{m>0} \sum_{n \leq 0}\left(n \mathrm{e}^{-i(m-n) t}-m \mathrm{e}^{i(m-n) t}\right) \\
= & \sum_{k>0} \sum_{m=1}^{k}(m-k) \mathrm{e}^{-i k t}-m \mathrm{e}^{i k t}=\sum_{k>0}-\frac{k(k-1)}{2} \mathrm{e}^{-i k t}-\frac{k(k+1)}{2} \mathrm{e}^{i k t}
\end{array}
$$




$$
\begin{aligned}
& =-\sum_{k} \frac{k(k-1)}{2} \mathrm{e}^{-i k t}=\pi \ddot{\delta}(t)+\pi i \dot{\delta}(t) . \\
i i i . \quad & -4 \pi^{2} \cdot L H S=\sum_{m>0} \sum_{n \leq 0}\left(m n \mathrm{e}^{-i(m-n) t}-m n \mathrm{e}^{i(m-n) t}\right) \\
& =\sum_{k>0} \sum_{m=1}^{k} m(m-k)\left(\mathrm{e}^{-i k t}-\mathrm{e}^{i k t}\right)=\sum_{k>0}-\frac{k^{3}-k}{6}\left(\mathrm{e}^{-i k t}-\mathrm{e}^{i k t}\right) \\
& =-\sum_{k} \frac{k^{3}-k}{6} \mathrm{e}^{-i k t}=\frac{1}{6}(2 \pi i \dddot{\delta}(t)+2 \pi i \dot{\delta}(t)) .
\end{aligned}
$$

Define

$$
\begin{aligned}
\tilde{\xi}^{i}(t) & \equiv \tilde{\xi}^{i}(q(t), \dot{q}(t))=\xi^{i}(q(t))-\xi^{0}(q(t)) \dot{q}^{i}(t), \\
\chi_{\xi j}^{>i}(t, s) & \equiv\left[p_{j}^{>}(t), \tilde{\xi}^{i}(s)\right] \\
& =\partial_{j} \tilde{\xi}^{i}(s) \delta^{>}(t-s)+\delta_{j}^{i} \xi^{0}(s) \dot{\delta}^{>}(t-s),
\end{aligned}
$$

and $\chi_{\xi j}^{\leq i}(t, s)$ analogously. Moreover, set $\chi_{\xi j}^{i}(t, s)=\chi_{\xi j}^{>i}(t, s)+\chi_{\xi j}^{\leq i}(t, s)$.

Lemma A.2 The expressions defined in (A.0) satisfy the following relations.

$$
\begin{aligned}
\partial_{i} \tilde{\xi}^{i}= & \partial_{\mu} \xi^{\mu}-\dot{\xi}^{0} \\
\partial_{j} \dot{\tilde{\xi}}^{i} \partial_{i} \tilde{\eta}^{j}= & \partial_{\nu} \dot{\xi}^{\mu} \partial_{\mu} \eta^{\nu}+\partial_{\nu} \xi^{0} \dot{q}^{\rho} \partial_{\rho} \dot{\eta}^{\nu}-\dot{q}^{\rho} \partial_{\rho} \dot{\xi}^{\mu} \partial_{\mu} \eta^{0}-\ddot{\xi}^{0} \dot{\eta}^{0} \\
& -\dot{\xi}^{0} \dot{q}^{\rho} \partial_{\rho} \dot{\eta}^{0}+\dot{q}^{\rho} \partial_{\rho} \dot{\xi}^{0} \dot{\eta}^{0}+\frac{d}{d t}\left(\dot{\xi}^{0} \dot{\eta}^{0}-\partial_{\nu} \xi^{0} \dot{\eta}^{\nu}\right) .
\end{aligned}
$$

Proof: We use that $\tilde{\xi}^{0} \equiv 0$. Eq. (A.8) thus equals

$$
\partial_{\mu} \tilde{\xi}^{\mu}=\partial_{\mu} \xi^{\mu}-\partial_{\mu} \xi^{0} \dot{q}^{\mu}
$$

whereas A.9 becomes

$$
\begin{aligned}
\partial_{\nu} \dot{\tilde{\xi}}^{\mu} \partial_{\mu} \tilde{\eta}^{\nu}= & \left(\partial_{\nu} \dot{\xi}^{\mu}-\partial_{\nu} \xi^{0} \ddot{q}^{\mu}-\partial_{\nu} \dot{\xi}^{0} \dot{q}^{\mu}\right)\left(\partial_{\mu} \eta^{\nu}-\partial_{\mu} \eta^{0} \dot{q}^{\nu}\right) \\
= & \partial_{\nu} \dot{\xi}^{\mu} \partial_{\mu} \eta^{\nu}-\partial_{\nu} \xi^{0}\left(\ddot{\eta}^{\nu}-\dot{q}^{\rho} \partial_{\rho} \dot{\eta}^{\nu}\right)-\partial_{\nu} \dot{\xi}^{0} \dot{\eta}^{\nu} \\
& -\dot{q}^{\rho} \partial_{\rho} \dot{\xi}^{\mu} \partial_{\mu} \eta^{0}+\dot{\xi}^{0}\left(\ddot{\eta}^{0}-\dot{q}^{\rho} \partial_{\rho} \dot{\eta}^{0}\right)+\dot{q}^{\rho} \partial_{\rho} \dot{\xi}^{0} \dot{\eta}^{0}
\end{aligned}
$$


Consider

$$
\begin{aligned}
\mathcal{L}_{\xi}^{0}=\int d t & : \xi^{\mu}(q(t)) p_{\mu}(t): \equiv \int d t\left(\tilde{\xi}^{i}(t) p_{i}^{\leq}(t)+p_{i}^{>}(t) \tilde{\xi}^{i}(t)\right) . \\
{\left[\mathcal{L}_{\xi}^{0}, \mathcal{L}_{\eta}^{0}\right]=} & \iint d s d t\left[\tilde{\xi}^{i}(s) p_{i}^{\leq}(s)+p_{i}^{>}(s) \tilde{\xi}^{i}(s),\right. \\
= & \iint d s d t\left\{\tilde{\eta}^{j}(t) p_{j}^{\leq}(t)+p_{j}^{>}(t) \tilde{\eta}^{j}(t)\right] \\
& \int \chi_{\eta i}^{\leq j}(s, t) p_{j}^{\leq}(t)-\tilde{\eta}^{j}(t) \chi_{\bar{\xi} j}^{\leq i}(t, s) p_{i}^{\leq}(s) \\
& +\tilde{\xi}^{i}(s) p_{j}^{>}(t) \chi_{\eta i}^{\leq j}(s, t)-\chi_{\xi j}^{>i}(t, s) \tilde{\eta}^{j}(t) p_{i}^{\leq}(s) \\
& +\chi_{\eta i}^{>j}(s, t) p_{j}^{\leq}(t) \tilde{\xi}^{i}(s)-p_{i}^{>}(s) \tilde{\eta}^{j}(t) \chi_{\xi_{j}}^{\leq i}(t, s) \\
& \left.-p_{i}^{>}(s) \chi_{\xi j}^{>i}(t, s) \tilde{\eta}^{j}(t)+p_{j}^{>}(t) \chi_{\eta i}^{>j}(s, t) \tilde{\xi}^{i}(s)\right\} .
\end{aligned}
$$

Of these eight terms, the third can be rewritten as

$$
p_{j}^{>}(t) \tilde{\xi}^{i}(s) \chi_{\eta i}^{\leq j}(s, t)-\chi_{\xi j}^{>i}(t, s) \chi_{\eta i}^{\leq j}(s, t)
$$

and the fifth as

$$
\chi_{\eta i}^{>j}(s, t) \tilde{\xi}^{i}(s) p_{j}^{\leq}(t)+\chi_{\eta i}^{>j}(s, t) \chi_{\bar{\xi} j}^{\leq i}(t, s) .
$$

Hence

$$
\begin{aligned}
& {\left[\mathcal{L}_{\xi}^{0}, \mathcal{L}_{\eta}^{0}\right]=\iint d s d t\left\{\tilde{\xi}^{i}(s) \chi_{\eta i}^{\leq j}(s, t) p_{j}^{\leq}(t)-\tilde{\eta}^{j}(t) \chi_{\xi j}^{\leq i}(t, s) p_{i}^{\leq}(s)\right.} \\
& \quad+p_{j}^{>}(t) \tilde{\xi}^{i}(s) \chi_{\eta i}^{\leq j}(s, t)-\tilde{\eta}^{j}(t) \chi_{\xi j}^{>i}(t, s) p_{i}^{\leq}(s) \\
& \quad+\tilde{\xi}^{i}(s) \chi_{\eta i}^{>j}(s, t) p_{j}^{\leq}(t)-p_{i}^{>}(s) \tilde{\eta}^{j}(t) \chi_{\overline{\xi j}}^{\leq i}(t, s) \\
& \left.\quad-p_{i}^{>}(s) \tilde{\eta}^{j}(t) \chi_{\xi j}^{>i}(t, s)+p_{j}^{>}(t) \tilde{\xi}^{i}(s) \chi_{\eta i}^{>j}(s, t)\right) \\
& \left.\quad-\chi_{\xi j}^{>i}(t, s) \chi_{\eta i}^{\leq j}(s, t)+\chi_{\eta i}^{>j}(s, t) \chi_{\xi j}^{\leq i}(t, s)\right\}
\end{aligned}
$$

The regular piece is

$$
\iint d s d t \tilde{\xi}^{i}(s) \chi_{\eta_{i}}^{j}(s, t) p_{j}^{\leq}(t)+p_{j}^{>}(t) \tilde{\xi}^{i}(s) \chi_{\eta_{i}}^{j}(s, t)-\xi \leftrightarrow \eta .
$$


We focus on the first term.

$$
\begin{aligned}
& \iint d s d t \tilde{\xi}^{i}(s) \chi_{\eta_{i}^{j}}(s, t) p_{j}^{\leq}(t)-\xi \leftrightarrow \eta \\
= & \iint d s d t \tilde{\xi}^{\mu}(s)\left(\partial_{\mu} \tilde{\eta}^{j}(t) \delta(s-t)+\eta^{0}(t) \delta_{\mu}^{j} \dot{\delta}(s-t)\right) p_{j}^{\leq}(t)-\xi \leftrightarrow \eta \\
= & \int\left\{\left(\widehat{\xi^{\mu} \partial_{\mu} \eta}\right)^{j}-\xi^{0}\left(\dot{\eta}^{j}-\dot{\eta}^{0} \dot{q}^{j}\right)-\dot{\tilde{\xi}}^{j} \eta^{0}\right\} p_{j}^{\leq}-\xi \leftrightarrow \eta,
\end{aligned}
$$

which equals $\mathcal{L}_{[\xi, \eta]}^{0}$. We again suppress the integration variable in single integrals, and write $\xi^{\mu}(s) \equiv \xi^{\mu}(q(s))$, etc. The extension $\operatorname{ext}_{0}(\xi, \eta)$ becomes

$$
\begin{aligned}
& \iint d s d t\left\{-\chi_{\xi j}^{>i}(t, s) \chi_{\eta i}^{\leq j}(s, t)+\chi_{\eta i}^{>j}(s, t) \chi_{\xi j}^{\leq i}(t, s)\right\} \\
= & -\iint d s d t\left\{\left(\partial_{j} \tilde{\xi}^{i}(s) \delta^{>}(t-s)+\delta_{j}^{i} \xi^{0}(s) \dot{\delta}^{>}(t-s)\right) \times\right. \\
& \left.\times\left(\partial_{i} \tilde{\eta}^{j}(t) \delta^{\leq}(s-t)+\delta_{i}^{j} \eta^{0}(t) \dot{\delta}^{\leq}(s-t)\right)\right\}-\xi \leftrightarrow \eta \\
= & -\iint d s d t\left\{\partial_{j} \tilde{\xi}^{i}(s) \partial_{i} \tilde{\eta}^{j}(t) \delta^{>}(t-s) \delta^{\leq}(s-t)\right. \\
& +\xi^{0}(s) \partial_{j} \tilde{\eta}^{j}(t) \dot{\delta}^{>}(t-s) \delta^{\leq}(s-t) \\
& +\partial_{i} \tilde{\xi}^{i}(s) \eta^{0}(t) \delta^{>}(t-s) \dot{\delta}^{\leq} \leq(s-t) \\
& \left.+\delta_{i}^{i} \xi^{0}(s) \eta^{0}(t) \dot{\delta}^{>}(t-s) \dot{\delta}^{\leq} \leq(s-t)\right\}-\xi \leftrightarrow \eta \\
= & \frac{1}{2 \pi i} \iint d s d t\left\{\partial_{j} \tilde{\xi}^{i}(s) \partial_{i} \tilde{\eta}^{j}(t) \dot{\delta}(t-s)\right. \\
& +\frac{1}{2} \xi^{0}(s) \partial_{j} \tilde{\eta}^{j}(t)(\ddot{\delta}(t-s)-i \dot{\delta}(t-s)) \\
& -\frac{1}{2} \partial_{i} \tilde{\xi}^{i}(s) \eta^{0}(t)(\ddot{\delta}(t-s)+i \dot{\delta}(t-s)) \\
& \left.-\frac{N-1}{6} \xi^{0}(s) \eta^{0}(t)(\dddot{\delta}(t-s)+\dot{\delta}(t-s))\right\} \\
= & \frac{1}{2 \pi i} \int\left\{\partial_{j} \dot{\tilde{\xi}}^{i} \partial_{i} \tilde{\eta}^{j}-\frac{1}{2} \dot{\xi}^{0} \partial_{j} \dot{\tilde{\eta}}^{j}+\frac{1}{2} \partial_{i} \dot{\tilde{\xi}}^{i} \dot{\eta}^{0}\right. \\
& \left.-\frac{N-1}{6}\left(-\ddot{\xi}^{0} \dot{\eta}^{0}+\dot{\xi}^{0} \eta^{0}\right)+\frac{i}{2}\left(-\dot{\xi}^{0} \partial_{j} \tilde{\eta}^{j}+\partial_{i} \tilde{\xi}^{i} \dot{\eta}^{0}\right)\right\},
\end{aligned}
$$

where we used Lemma A.1 and the fact that $\delta_{i}^{i}=N-1$. Now consider the full algebra. The regular piece follows from the following calculation.

$$
\int\left(\xi^{\mu} \partial_{\mu} \eta^{\nu}-\xi^{0} \dot{\eta}^{\nu}\right) p_{\nu}+\iint d s d t \xi^{\mu}(s) \eta^{\nu}(t) \delta_{\mu}^{0} p_{\nu}(s) \dot{\delta}(s-t)
$$




$$
\begin{aligned}
& +\int\left(\xi^{\mu} \partial_{\mu} \eta^{0}-\xi^{0} \dot{\eta}^{0}\right) L+\iint d s d t \xi^{0}(s) \eta^{0}(t) L(s) \dot{\delta}(s-t) \\
& +\int\left\{\left(\xi^{\mu} \partial_{\mu} \partial_{\sigma} \eta^{\tau}-\xi^{0} \partial_{\sigma} \dot{\eta}^{\tau}\right) T_{\tau}^{\sigma}+\partial_{\mu} \xi^{\nu} \partial_{\sigma} \eta^{\tau} \delta_{\nu}^{\sigma} T_{\tau}^{\mu}\right\} \\
& +\iint d s d t \xi^{0}(s) \partial_{\sigma} \eta^{\tau}(t) T_{\tau}^{\sigma}(s) \dot{\delta}(s-t)-\xi \leftrightarrow \eta \\
& =\int[\xi, \eta]^{\nu} p_{\nu}+[\xi, \eta]^{0} L+\partial_{\mu}[\xi, \eta]^{\nu} T_{\nu}^{\mu},
\end{aligned}
$$

and the full extension is

$$
\begin{aligned}
\operatorname{ext}(\xi, \eta)= & \operatorname{ext}_{0}(\xi, \eta)+\iint d s d t\left\{\frac{c}{24 \pi i} \xi^{0}(s) \eta^{0}(t)(\dddot{\delta}(s-t)+\dot{\delta}(s-t))\right. \\
& +\frac{k_{0}}{4 \pi i}\left(\xi^{0}(s) \partial_{\nu} \eta^{\nu}(t)-\partial_{\mu} \xi^{\mu}(s) \eta^{0}(t)\right) \ddot{\delta}(s-t) \\
& \left.-\partial_{\sigma} \xi^{\mu}(s) \partial_{\tau} \eta^{\nu}(t)\left(\frac{k_{1}}{2 \pi i} \delta_{\nu}^{\sigma} \delta_{\mu}^{\tau}+\frac{k_{2}}{2 \pi i} \delta_{\mu}^{\sigma} \delta_{\nu}^{\tau}\right) \dot{\delta}(s-t)\right\} \\
= & \operatorname{ext}_{0}(\xi, \eta)+\frac{1}{2 \pi i} \int\left\{\frac{c}{12}\left(\ddot{\xi}^{0} \dot{\eta}^{0}-\dot{\xi}^{0} \eta^{0}\right)\right. \\
& \left.+\frac{k_{0}}{2}\left(-\dot{\xi}^{0} \partial_{\nu} \dot{\eta}^{\nu}+\dot{\eta}^{0} \partial_{\mu} \dot{\xi}^{\mu}\right)+k_{1} \partial_{\nu} \dot{\xi}^{\mu} \partial_{\mu} \eta^{\nu}+k_{2} \partial_{\mu} \dot{\xi}^{\mu} \partial_{\nu} \eta^{\nu}\right\}
\end{aligned}
$$

The result now follows by means of lemma A.2.

$$
\begin{aligned}
& \operatorname{ext}(\xi, \eta)=\frac{1}{2 \pi i} \int d t\left\{\left(1+k_{1}\right) \partial_{\nu} \dot{\xi}^{\mu} \partial_{\mu} \eta^{\nu}+k_{2} \partial_{\mu} \dot{\xi}^{\mu} \partial_{\nu} \eta^{\nu}\right. \\
& \quad+\partial_{\nu} \xi^{0} \dot{q}^{\rho} \partial_{\rho} \dot{\eta}^{\nu}-\dot{q}^{\rho} \partial_{\rho} \dot{\xi}^{\mu} \partial_{\mu} \eta^{0}-\dot{\xi}^{0} \dot{q}^{\rho} \partial_{\rho} \dot{\eta}^{0}+\dot{q}^{\rho} \partial_{\rho} \dot{\xi}^{0} \dot{\eta}^{0} \\
& \quad+\frac{1+k_{0}}{2}\left(\partial_{\mu} \dot{\xi}^{\mu} \dot{\eta}^{0}-\dot{\xi}^{0} \partial_{\nu} \dot{\eta}^{\nu}\right)-\left(2-\frac{c+2(N-1)}{12}\right) \ddot{\xi}^{0} \dot{\eta}^{0} \\
& \left.\quad-\frac{c+2(N-1)}{12} \dot{\xi}^{0} \eta^{0}+\frac{i}{2}\left(\partial_{\mu} \xi^{\mu} \dot{\eta}^{0}-\dot{\xi}^{0} \partial_{\nu} \eta^{\nu}\right)\right\}
\end{aligned}
$$

where $\dot{f}=\dot{q}^{\rho} \partial_{\rho} f$. As a consistency check we note that the extension satisfies $\operatorname{ext}(\eta, \xi)=-\operatorname{ext}(\xi, \eta)$.

To calculate the remaining brackets is a straightforward task. Note that normal ordering is irrelevant here, because $S_{n}^{\nu_{1} . . \nu_{n}}$ and $R_{n}^{\rho \mid \nu_{1} . . \nu_{n}}$ depend on $q^{\mu}$ only whereas $\mathcal{L}_{\xi}$ depends only linearly on $p_{\nu}$.

Note added. A. Dzhumadil'daev has explained his results [3], which I had slightly misunderstood. The Rao-Moody cocycle $c_{1}$ is included in his list; it is equivalent to his cocycle $\psi_{4}^{W}$, with coefficients in $\Omega_{\text {DeRham }}^{1} / B_{\text {DeRham }}^{1} \cong$ $B_{\text {DeRham }}^{2} \oplus H_{\text {DeRham }}^{1}$. Similarly, $c_{2}$ is his $\psi_{3}^{W} . H_{\text {DeRham }}^{1}$ is an $N$-dimensional 
trivial diff $f(N)$ module; setting it to zero gives the substitution (2.5). The closedness condition $S_{1}^{\rho}\left(\partial_{\rho} f\right)=0$ can be lifted for the cocycle $c_{2}$ (but not for $c_{1}$ ). One then obtains $\psi_{1}^{W}$, first discovered in [7]. Dzhumadil'daev considered extensions by modules of tensor fields, not necessarily irreducible. $c_{3}$ and $c_{4}$ are not included in his list, because they are extensions by other types of modules.

\section{References}

[1] Berman, S. and Y. Billig, Irreducible representations for toroidal Lie algebras, preprint (1998).

[2] Billig, Y., Principal vertex operator representations for toroidal Lie algebras, J. Math. Phys. 7, 3844-3864 (1998).

[3] Dzhumadildaev A., Virasoro type Lie algebras and deformations, Z. Phys. C 72, 509-517 (1996).

[4] Eswara Rao, S., R.V. Moody and T. Yokonuma, Lie algebras and Weyl groups arising from vertex operator representations, Nova J. of Algebra and Geometry 1, 15-57 (1992).

[5] Eswara Rao, S. and R.V. Moody, Vertex representations for $N$-toroidal Lie algebras and a generalization of the Virasoro algebra, Commun. Math. Phys. 159, 239-264 (1994).

[6] Fabbri, M. and R.V. Moody, Irreducible representations of Virasosotoroidal Lie algebras, Commun. Math. Phys. 159, 1-13 (1994).

[7] Larsson, T.A., Multi-dimensional Virasoro algebra, Phys. Lett. A 231, 94-96 (1989).

[8] Larsson, T.A., Central and non-central extensions of multi-graded Lie algebras, J. Phys. A. 25, 1177-1184 (1992).

[9] Larsson, T.A., Fock representations of non-centrally extended superdiffeomorphism algebras, physics/9710022 (1997).

[10] Moody, R.V., S. Eswara Rao and T. Yokonoma, Toroidal Lie algebras and vertex representations, Geom. Ded. 35, 283-307 (1990). 